\title{
Evolutionary phases of merging clusters as seen by LOFAR
}

\author{
A. Wilber ${ }^{1}$, M. Brüggen ${ }^{1}$, A. Bonafede ${ }^{1,2,3}$, D. Rafferty ${ }^{1}$, T. W. Shimwell ${ }^{4,5}$, R. J. van Weeren ${ }^{5}$, H. Akamatsu ${ }^{6}$, \\ A. Botteon ${ }^{2,3}$, F. Savini ${ }^{1}$, H. Intema ${ }^{5}$, L. Heino ${ }^{1}$, V. Cuciti ${ }^{2,3}$, R. Cassano ${ }^{2}$, G. Brunetti ${ }^{2}$, \\ H. J. A. Röttgering ${ }^{5}$, and F. de Gasperin ${ }^{1}$ \\ 1 Hamburger Sternwarte, Universität Hamburg, Gojenbergsweg 112, 21029 Hamburg, Germany \\ e-mail: amanda.wilber@hs.uni-hamburg.de \\ 2 Dipartimento di Fisica e Astronomia, Università di Bologna, Via P. Gobetti 93/2, 40129 Bologna, Italy \\ 3 INAF/Istituto di Radioastronomia, Via P. Gobetti 101, 40129 Bologna, Italy \\ 4 ASTRON, The Netherlands Institute for Radio Astronomy, Postbus 2, 7990 AA Dwingeloo, The Netherlands \\ 5 Leiden Observatory, Leiden University, PO Box 9513, 2300 RA Leiden, The Netherlands \\ ${ }^{6}$ SRON Netherlands Institute for Space Research, Utrecht, The Netherlands
}

Received 16 July 2018 / Accepted 22 August 2018

\section{ABSTRACT}

\begin{abstract}
Massive, merging galaxy clusters often host giant, diffuse radio sources that arise from shocks and turbulence; hence, radio observations can be useful for determining the merger state of a cluster. In preparation for a larger study, we selected three clusters - Abell 1319, Abell 1314, and RXC J1501.3+4220 (Z7215) - making use of the new LOFAR Two-Metre Sky Survey (LoTSS) at 120-168 MHz, and together with archival data, show that these clusters appear to be in pre-merging, merging, and post-merging states, respectively. We argue that Abell 1319 is likely in its pre-merging phase, where three separate cluster components are about to merge. There are no radio haloes nor radio relics detected in this system. Abell 1314 is a highly disturbed, low-mass cluster that is likely in the process of merging. This low-mass system does not show a radio halo, however, we argue that the merger activates mechanisms that cause electron re-acceleration in the large $800 \mathrm{kpc}$ radio tail associated with IC 711. In the cluster Z7215 we discover diffuse radio emission at the cluster centre, and we classify this emission as a radio halo, although it is dimmer and smaller than expected by the radio halo power versus cluster mass correlation. We suggest that the disturbed cluster Z7215 is in its post-merging phase. Systematic studies of this kind over a larger sample of clusters observed with LoTSS will help to constrain the timescales involved in turbulent re-acceleration and the subsequent energy losses of the underlying electrons.
\end{abstract}

Key words. galaxies: clusters: general - radio continuum: galaxies - galaxies: clusters: individual: RXC J1501.3+4220 galaxies: clusters: individual: Abell 1314 - galaxies: clusters: individual: Abell 1319 - galaxies: clusters: intracluster medium

\section{Introduction}

In the process of the hierarchical formation of structure in the Universe, clusters of galaxies merge to form more massive clusters (see Kravtsov \& Borgani 2012, for a review). Cluster mergers are violent phenomena, releasing large amounts of energy $\left(\sim 10^{63} \mathrm{erg}\right)$ into the intra-cluster medium (ICM). A merging system of galaxy clusters goes through three main evolutionary phases in a period of about one gigayear: a pre-merging phase in which the galaxy clusters have begun their infall and the intracluster media begin to interact, a merging phase in which core passage occurs, and a post-merging phase in which the ICM relaxes as shocks and turbulence begin to dissipate. The avenues of this energy release are not well understood and concern fundamental properties of dilute, magnetised plasmas.

Customarily, X-ray observations are used to classify clusters of galaxies as either merging or non-merging. Merging clusters are identified by morphologically disturbed thermal Bremsstrahlung emission from the ICM while non-merging clusters typically have a relaxed morphology and compact cool cores (e.g. Molendi \& Pizzolato 2001). Several disturbed, massive clusters have been observed to host cluster-scale sources of radio synchrotron emission. Such sources come in two forms: radio haloes, which are diffuse, unpolarised sources that fill most of the inner volume of clusters; and radio relics, which are elongated, polarised sources found in the cluster outskirts. Radio haloes and relics typically exhibit a steep synchrotron spectrum $\left(\alpha<-1\right.$ where $S \propto v^{\alpha}$ ), have low surface brightness, and are usually of the order of $500 \mathrm{kpc}$ to $1 \mathrm{Mpc}$ in size (see Feretti et al. 2012 , for a review). The origins of radio haloes and relics and the acceleration mechanisms of the cosmic-ray electrons that power these sources are still under debate (see Brunetti \& Jones 2014, for a review).

Radio haloes are thought to be powered by turbulent reacceleration of electrons. In this scenario, turbulence generated by a cluster merger re-accelerates mildly relativistic electrons in situ (Petrosian 2001; Brunetti et al. 2001, 2008). These cosmicray electrons then produce synchrotron radiation at radio frequencies within the cluster magnetic field, which is of the order of a few $\mu \mathrm{G}$ (Carilli \& Taylor 2002). The specific radio luminosities of haloes observed at $1.4 \mathrm{GHz}$ range between about $10^{23}\left[\mathrm{~W} \mathrm{~Hz}^{-1}\right]$ and $10^{25}\left[\mathrm{~W} \mathrm{~Hz}^{-1}\right]$. Haloes usually occur in isolation with the exception of the double radio haloes in the pre-merging cluster pair Abell 399-401 (Murgia et al. 2010) and Abell 1758N-A1758S (Botteon et al. 2018). Radio haloes have a smooth brightness distribution that roughly follows the 
distribution of the thermal ICM; however, some haloes with irregular morphologies have been found (e.g. Giacintucci et al. 2009; Giovannini et al. 2011). Some radio haloes exhibit exceptionally steep spectra, where $\alpha \sim-2$. These ultra-steep spectrum radio haloes (USSRHs) may originate from low-mass mergers where the energy budget for turbulent re-acceleration is smaller, or when the radio halo starts to fade at a later evolutionary stage. A prominent example of such a USSRH is that discovered in Abell 521 (Brunetti et al. 2008; Dallacasa et al. 2009).

Questions remain concerning the particle acceleration efficiency of this turbulence, the source of mildly relativistic seed electrons that must already fill the ICM before they are reaccelerated, and the origin and amplification of the cluster magnetic field. A handful of unusual clusters offer interesting insights into these questions. For example, cluster-scale diffuse radio emission has been seen in some cool-core, relaxed clusters (e.g. Bonafede et al. 2014). Furthermore, not all merging systems show radio haloes and only upper limits for the diffuse radio flux are known (e.g. Brunetti et al. 2007). The fraction of clusters that host radio haloes provide fundamental input for theory (Cassano et al. 2010). Radio relics are more direct indicators of merger activity as they are thought to trace merger-induced shocks. The position of the radio relics with respect to other cluster components, such as galaxies and dark matter, can help to reconstruct the geometry of the merger (e.g. Golovich et al. 2018).

Another way to assess the merging state of clusters is to examine the interplay between the radio lobes of active galactic nuclei (AGNs) and the ICM. Active radio galaxies are suspected to contribute a population of relativistic electrons to their surrounding medium, which may provide a portion of the seed electrons needed to explain diffuse ICM radio sources. An example of such is the connection between a radio relic and a radio galaxy in Abell 3411-3412 (van Weeren et al. 2017). Extended radio galaxies, with sizes $\geq 700 \mathrm{kpc}$, may serve as large suppliers of these seed electrons (e.g. Wilber et al. 2018a). Injection of seed electrons from AGNs can also be studied by looking at remnant radio galaxies, which are radio galaxies in the phase after which nuclear activity has ceased (e.g. Brienza et al. 2017; Brunetti \& Jones 2014). For radio galaxies with intermittent activity, this remnant, or fossil, emission may appear as fading lobes that are visibly detached from an active AGN core. Radio galaxies moving through the cluster environment may also experience ram pressure that results in a bent-tail (BT) morphology (Miley 1980). Studying the output of radio AGNs within cluster environments is crucial to understanding the characteristics of the ICM.

In recent years, the LOw Frequency ARray (LOFAR) has been a key instrument used to study faint cluster-scale radio sources associated with merging galaxy clusters. The LOFAR instrument is a low-frequency radio interferometer with a compact core in the Netherlands and stations presently located in six other European countries (van Haarlem et al. 2013). The LOFAR Two-Metre Sky Survey (LoTSS; Shimwell et al. 2017) is a new low-frequency survey aiming to map the entire northern sky. When completed, it will be two orders of magnitude deeper in point-source sensitivity and one order of magnitude higher in resolution than any current very large radio survey at this frequency regime (Shimwell et al. 2019). The first 600 pointings ( $20 \%$ of the total) of this survey have recently been observed, covering the $120-168 \mathrm{MHz}$ band using high-band antennas (HBAs). The LOFAR instrument has already yielded valuable insights into radio haloes. These include the observation of intricate and filamentary structures embedded in a radio halo (Shimwell et al. 2016), the discovery of a new USSRH
(Wilber et al. 2018a), the detection of extended radio emission in two cool-core clusters (Savini et al. 2019), and the observation of a tentative bridge of emission connecting two radio haloes (Botteon et al. 2018).

Theoretical studies have predicted that LOFAR will detect many more radio haloes, especially haloes with ultra-steep spectra $(\alpha \leq-1.5)$ since these objects appear brighter at lower frequencies (e.g. Brunetti et al. 2008; Cassano et al. 2012). Studies of galaxy clusters made with observations from the Giant Metrewave Radio Telescope (GMRT), operating in a frequency range of $150 \mathrm{MHz}$ to $1.4 \mathrm{GHz}$, have already revealed that radio haloes can change in brightness and morphology depending upon the observing frequency and that some radio haloes are only visible at lower frequencies (e.g. Venturi et al. 2007, 2008; Kale et al. 2015).

We report on our search for cluster-scale diffuse radio emission associated with three merging cluster systems: Abell 1314, Abell 1319, and RXC J1501.3+4220 (referred to as Z7215 hereafter). In preparation for a larger study, which will consist of LOFAR observations of many merging clusters, we selected these three clusters since they were already covered by LoTSS and appeared to host potential diffuse emission upon first inspection of preliminary images. These three clusters represent a small set of lower mass mergers that are likely at different evolutionary stages. We aim to determine the nature of the radio emission in these clusters and relate this to their merger phase. Hereafter we use the cosmology $\mathrm{H}_{0}=69.6, \Omega_{m}=0.286$, and $\Omega_{\Lambda}=0.714$.

\section{Methods}

\subsection{LOFAR observations and data reduction}

The $8 \mathrm{~h}$ LOFAR observations we report in this paper were made as part of LoTSS (Shimwell et al. 2017) over a frequency range of $120-168 \mathrm{MHz}$ using the Dutch HBAs. The data reduction steps for these data are identical to the steps detailed in Wilber et al. (2018a,b), but are also reiterated below.

\subsubsection{Prefactor}

Prefactor $^{1}$ is a package containing automated pipelines called Pre-Facet-Calibration and Initial-Subtract. Pre-FacetCalibration compresses and averages the original data and performs the initial direction-independent calibration (see de Gasperin et al. 2019, for details). In this step a flux calibrator (observed at the beginning and end of the target observation) was used to compute amplitude gain solutions, station clock offsets, station phase offsets, and station differential total electron content (dTEC). Amplitude gain solutions and corrections for clock and phase offsets were then transferred to the target field data. An initial phase calibration was also performed using a global sky model from the TIFR GMRT Sky Survey (TGSS) at $150 \mathrm{MHz}$ (Intema et al. 2017). The calibrator 3C196, which is a bright quasar (66 Jy at $159 \mathrm{MHz}$ according to the Scaife \& Heald 2012 absolute flux scale) was used for the observations of all three of our selected clusters. After the direction-independent calibration was completed, preliminary imaging was carried out via the Initial-Subtract pipeline. The full wide-field of the calibrated target data was imaged in high and low resolution using WSClean (Offringa et al. 2014). These full-field images were used to model and subtract all sources in preparation for direction-dependent calibration.

\footnotetext{
1 https://github.com/lofar-astron/prefactor
} 


\subsubsection{FACTOR}

Direction-dependent calibration for our LoTSS data was carried out through the facet calibration technique (van Weeren et al. 2016). This method of calibration is executed via the FACTOR ${ }^{2}$ software package. FACTOR tesselated the full target field into several smaller portions of sky called facets, where each facet is automatically chosen to be centred on a bright compact source to be used as a facet calibrator. The TEC, phase, and amplitude solutions were computed from the facet calibrator and applied to all the sources in that facet. Facets were processed in order of brightness, and all facet sources were subtracted from the $u v$-data before processing the next facet. This method reduced the effective noise in subsequent calibration steps. The target facet, containing the cluster of interest, was appointed as the last facet in the processing list, such that all other nearby and bright sources were already calibrated and subtracted. The calibration region for the target facet was chosen either as the cluster centre, containing multiple sources, or as a single bright, compact source near the cluster centre. More details on facet calibration are provided in van Weeren et al. (2016), Shimwell et al. (2016), and Williams et al. (2016).

\subsection{LOFAR imaging}

The final FACTOR-calibrated data of all three clusters were imaged and analysed with CASA tools (Common Astronomy Software Applications; McMullin et al. 2007). To lower the resolution and increase the sensitivity to diffuse radio emission on large scales, we used the CASA task CLEAN and chose an increased outer $u v$-taper of up to 30 arcsec and Briggs' robust values of -0.25 or 0 . Final images made in CASA were also corrected for the LOFAR station beam. The error in our flux density measurements are assumed to be $10 \%$, which was determined by comparing flux densities of several sources in our LOFAR map to the same sources in TGSS (Intema et al. 2017) and the 7C survey (Hales et al. 2007). In the following subsections we describe the basic steps of data manipulation and imaging that were made on our LOFAR measurement sets.

\subsubsection{Subtraction of compact sources}

To accurately measure diffuse radio emission at the cluster centre, we used an image made after subtracting compact radio sources. Compact sources are usually found within the vicinity of the cluster centre and are typically associated with AGN. Hence, the scale of the compact emission we wish to subtract is of the order of $<200 \mathrm{kpc}$. A compact-source image was made using the task CLEAN in CASA by selecting a specific $u v$-range that corresponds to emission on a projected scale of $>200 \mathrm{kpc}$. Based on the redshift of the individual clusters, the $u v$-cut was made at 800 $\lambda$ for Abell 1314 and 4500 $\lambda$ for Abell 1319 and $\mathrm{Z7215}$. The model components from this compact-source image were then subtracted from the measurement set using tasks FTW and UVSUB. We then re-imaged the source-subtracted data set with a $u v$-range of $>80 \lambda$, and used an increased outer $u v$-taper of 30 arcsec to bring out diffuse emission.

If diffuse emission was present at the cluster centre, we measured the flux density of this diffuse emission contained within $2 \sigma$ or $3 \sigma$ contours. From this flux density we calculated a power at $144 \mathrm{MHz}$ considering the redshift of the cluster, and we extrapolated this power to $1.4 \mathrm{GHz}$ with a spectral index of $\alpha=-1.3$. This power at $1.4 \mathrm{GHz}$ is used to give a relative com-

\footnotetext{
2 http://www.astron.nl/citt/facet-doc/
}

parison to the power in the correlation for radio halo power versus cluster mass (referred to hereafter as the $P-M$ correlation) from Cassano et al. (2013).

\subsubsection{Injection of mock halo}

For the massive, merging cluster Z7215, we followed a previously used method of injecting a fake radio halo into the visibilities. This method has been typically used to compute upper limits to the diffuse emission of clusters without radio haloes (e.g. Venturi et al. 2008; Kale et al. 2013, 2015; Bonafede et al. 2017). These studies showed that the flux density of the injected radio halo is only partly recovered and this effect becomes relatively more important for fainter haloes. Since we detected faint diffuse emission at the centre of Z7215 (see Sect. 3.3) we used the injection procedure to evaluate the effect of such losses in the measurement of this diffuse flux (e.g. Brunetti et al. 2007; Venturi et al. 2008; Bonafede et al. 2017; Cuciti et al. 2018). Although Abell 1314 is a highly disturbed cluster (as seen by X-ray observations), detectable emission is not expected in such a low-mass system $\left(M_{500}<10^{14} M_{\odot}\right)$ owing to energy limitations (e.g. Cassano \& Brunetti 2005; Cassano et al. 2006; Cassano 2010; Bernardi et al. 2016). Therefore, we decided not to implement halo injection for Abell 1314.

Following these methods, we simulated a radio halo by injecting a radio source into the $u v$ data that has a central brightness $I_{0}$ and an $e$-folding radius of $r_{e}$ (Murgia et al. 2009). The length scale, $r_{e}$, is defined as the radius at which the brightness of a radio halo drops to $I_{0} / e$, and measuring this is independent of the sensitivity of the radio images ${ }^{3}$. The model of this mock radio halo was Fourier transformed into the visibility data (MODEL_DATA column), taking into account the w-projection parameter (Cornwell et al. 2005). A relatively empty region near the cluster centre, void of bright sources and artefacts, was chosen to host the injected flux. The data set was then re-imaged with an outer $u v$-taper of 30 arcsec. We considered the injected halo detected when it was recovered above $2 \sigma$ contours with a diameter of roughly $3 r_{e}$.

\subsection{Supplementary observations}

\subsubsection{GMRT observations of Abell 1314}

Archival GMRT data at 235 and $610 \mathrm{MHz}$ (Obs ID: 7909) centred on the cluster Abell 1314 were obtained and reprocessed with the SPAM pipeline (see Intema et al. 2017, for details). We used the $610 \mathrm{MHz}$ data with our LOFAR data at $144 \mathrm{MHz}$ to produce a spectral index map of the sources in Abell 1314 ${ }^{4}$. To produce the spectral index map, we imaged the cluster at both frequencies using the same clean settings in CASA clean: uvrange $>200 \lambda$, uniform weighting, and a $u v$-taper of 40 arcsec. We re-gridded the LOFAR map to the $610 \mathrm{MHz}$ GMRT map and smoothed the images to give the same beam size ( $47 \operatorname{arcsec} \times$ $35 \operatorname{arcsec})$.

\subsubsection{X-ray observations}

Shallow XMM-Newton observations (Obs ID: 0149900201 and 0402250201, raw exposure times $=18$ and $27.2 \mathrm{ks}$, respectively)

\footnotetext{
3 Bonafede et al. (2017) compared the values of RH and re found by Cassano et al. (2007) and Murgia et al. (2009) for the eight clusters in common in their samples and found that the median value of the ratio $R_{\mathrm{H}} / r_{e}$ is 2.6 .

4 The $235 \mathrm{MHz}$ image was not useful for our multi-frequency analysis.
} 
were used to characterise the thermal ICM emission in Abell 1314 and Z7215, respectively. The SAS v15.0 and the built-in extended source analysis software (ESAS) were used to process and calibrate the data obtained with the XMM-Newton European Photon Imaging Camera (EPIC). Following a standard procedure, the raw data were created by emchain, and the light curves were extracted and screened for time-variable background components by the mos-filter, pn-filter task. The total exposure time after screening was $16.9 \mathrm{ks}, 17.0 \mathrm{ks}$, and $12.9 \mathrm{ks}$ for MOS1, MOS2, and pn, respectively, for the Abell 1314 (0149900201) observation. The total exposure time after screening was $25.6 \mathrm{ks}$, $25.4 \mathrm{ks}$, and $21.6 \mathrm{ks}$ for MOS1, MOS2, and pn, respectively, for the Z7215 (0402250201) observation.

Abell 1319 was observed with Chandra ACIS-S for $9.91 \mathrm{ks}$ (Obs ID: 11760) and Z7215 was observed with the Chandra ACIS-I VFAINT mode for $13 \mathrm{ks}$ (Obs ID: 7899). We processed the Chandra data following Vikhlinin et al. $(2005)^{5}$. The background was examined in the $0.5-7 \mathrm{keV}$ band to search for high proton flares. For the final exposure corrected image we used a binning of 1 arcsec pixel ${ }^{-1}$. More details are provided in Vikhlinin et al. (2005).

By analysing the surface brightness of the X-ray thermal emission of the ICM, we computed morphological parameters indicative of the merging status of the cluster. The centroid shift parameter, $w$, is defined as the projected separation between the peak and centroid of the X-ray surface brightness distribution when the aperture used to compute this parameter decreases from the aperture radius, $R_{\text {ap }}=500 \mathrm{kpc}$, to smaller radii (Böhringer et al. 2010). The concentration parameter, $c$, is defined as the ratio of the X-ray surface brightness within a radius of $100 \mathrm{kpc}$ over $\mathrm{X}$-ray surface brightness within a radius of $500 \mathrm{kpc}$ (Santos et al. 2008). The $c-w$ morphological diagram adapted from Cuciti et al. (2015) is shown in Fig. 1. The parameters $c$ and $w$ are anti-correlated; relaxed clusters lie on the top left region and merging clusters lie on the bottom right region. The boundary lines are taken from Cassano et al. (2010) and defined at $w \sim 0.012$ and $c \sim 0.2$, such that larger values of $w$ and smaller values of $c$ indicate a disturbed, merging cluster (Cassano et al. 2016). Our computed values for $w$ and $c$ for each cluster are listed in Table 1 and shown in the diagram in Fig. 1

\section{Results}

Abell 1319 and Z7215 were detected by the Planck satellite (Planck Collaboration XXIX 2014) via the Sunyaev-Zel'dovich (SZ) effect and have SZ-derived mass estimates (listed in Table 1). Abell 1314 was not detected by Planck, but the most recent mass estimate of Abell 1314, from MCXC (MetaCatalogue X-ray galaxy clusters; Piffaretti et al. 2011), is $M_{500}=$ $0.4608 \times 10^{14} M_{\odot}$, suggesting that this cluster has a very low mass. We report new radio emission detected by LOFAR in the low-mass cluster Abell 1314, and we derive a mass estimate from XMM-Newton X-ray observations. We also report new diffuse radio emission detected by LOFAR in the intermediatemass cluster Z7215. Table 1 lists the individual details for our three selected clusters. In this paper we hypothesise the merging status of each cluster based on a joint radio-X-ray analysis and suggest that each cluster represents one of the three evolutionary phases of merging systems: Abell 1319 in a pre-merging phase, Abell 1314 in a merging phase, and Z7215 in a post-merging phase.

\footnotetext{
5 We used CIAO v4.6 and CALDB v4.7.2.
}

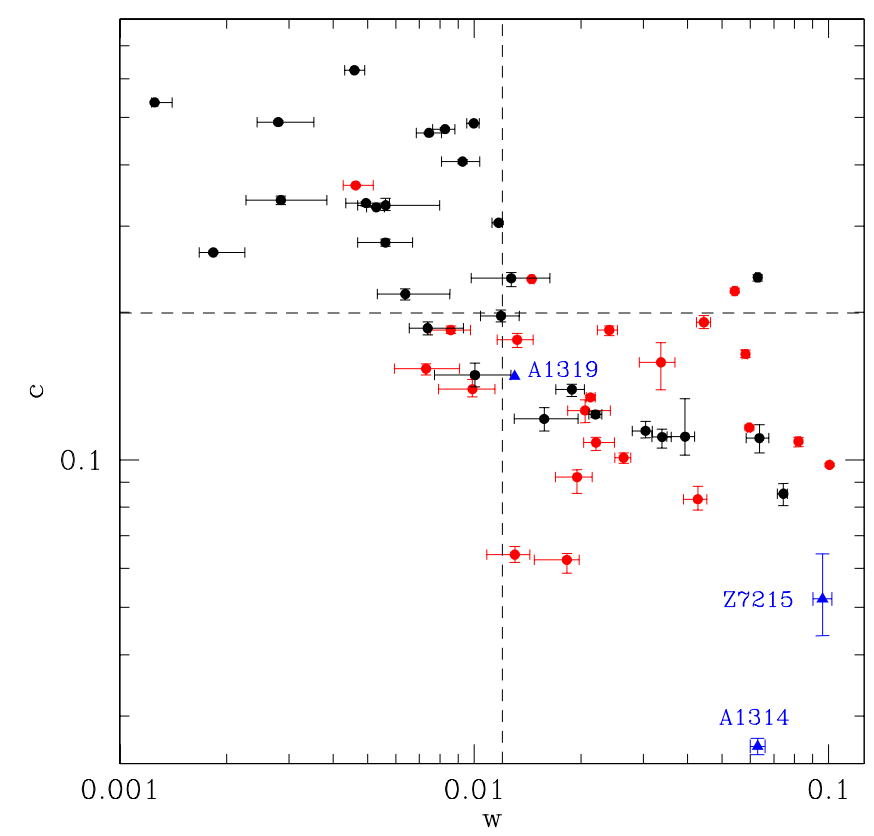

Fig. 1. Sample of clusters with masses $>6 \times 10^{14} M_{\odot}$ plotted by their concentration parameter $c$ vs. their centroid shift $w$ from Cuciti et al. (2015). Red dots indicate clusters hosting radio haloes and black dots indicate clusters without radio haloes. The three blue points represent Abell 1314, Abell 1319, and Z7215. The $w$ and $c$ parameters of all clusters are computed from Chandra X-ray images, except for Abell 1314 and Z7215, which have parameters computed from XMM-Newton images. Our selected clusters have masses lower than the sample from Cuciti et al. (2015).

\subsection{Abell 1319: Example of a pre-merging system}

In Fig. 2 we present an overlay of Chandra X-ray and LOFAR radio emission on a Sloan Digitial Sky Survey (SDSS) optical image of the cluster Abell 1319. This image of the cluster shows three ICM components. The main component is Abell 1319-A, and the two additional ICM components, to the northwest (Abell 1319-B) and southwest (Abell 1319-C), show coincident galaxies with spectroscopic redshifts (from the SDSS database) similar to that of the galaxies in Abell 1319-A.

In Fig. 3 Planck contours are overlaid on a X-ray image of Abell 1319 from ROSAT. All three ICM clumps are included in the Planck contours. Therefore, it is likely that the SZ-derived mass-estimate from Planck $\left(M_{500}=4.79_{-0.49}^{+0.51} \times 10^{14} M_{\odot}\right)$ is the mass of the total system, including all three ICM components. Although the Chandra image shows the southwest clump, Abell 1319-C, as being the most faint, ROSAT shows this component to be the brightest of the three, but this effect could be due to differences in the pointing and exposure times of the two separate observations. Abell 1319-C also lies near the CCD edge of the Chandra observation. We did not estimate the mass values of each individual cluster component.

Our LOFAR image of this multi-component system shows compact radio emission at the centre of each ICM. These are likely to be the radio AGN of bright central galaxies (BCGs). The optical overlay shows that the galaxies coincident with these compact central radio sources are all at similar redshifts. A more extended radio galaxy is seen on the eastern edge of Abell 1319-C. This emission is likely a BT radio galaxy from within the cluster (north) and a background radio galaxy (south) appearing in projection to be one extended source. After compactsource subtraction and uv-tapering to lower the resolution of 
Table 1. Our selected clusters observed by LOFAR.

\begin{tabular}{|c|c|c|c|c|c|c|c|c|}
\hline $\begin{array}{c}\text { Cluster name } \\
\text { (Obs ID) }\end{array}$ & $\begin{array}{c}\text { RA, Dec } \\
\text { J2000 }\end{array}$ & $\begin{array}{c}\text { Redshift } \\
z\end{array}$ & $\begin{array}{c}\text { Scale } \\
\mathrm{kpc} \operatorname{arcsec}^{-1}\end{array}$ & $\begin{array}{c}\text { Mass } \\
\left(10^{14} M_{\odot}\right)\end{array}$ & $\begin{array}{c}\text { Dynamics } \\
w_{500 \mathrm{kpc}}, c_{100 \mathrm{kpc}}\end{array}$ & $\begin{array}{c}\text { Halo } P_{1.4 \text { corr }} \\
\log _{10}\left(\mathrm{~W} \mathrm{~Hz}^{-1}\right)\end{array}$ & $\begin{array}{c}R_{\mathrm{H} \text { corr }} \\
(\mathrm{kpc})\end{array}$ & $\begin{array}{r}r_{e} \\
(\mathrm{kpc}) \\
\end{array}$ \\
\hline $\begin{array}{l}\text { Abell } 1314 \\
\text { (L229509) }\end{array}$ & $\begin{array}{l}11^{\mathrm{h}} 34^{\mathrm{m}} 50.5^{\mathrm{s}} \\
+49^{\mathrm{d}} 03^{\mathrm{m}} 28^{\mathrm{s}}\end{array}$ & 0.0335 & 0.672 & $\begin{array}{l}0.68^{a} \\
\text { Low }\end{array}$ & $\begin{array}{c}0.063 \pm 0.003 \\
0.026 \pm 0.01\end{array}$ & N/A & N/A & N/A \\
\hline $\begin{array}{l}\text { Abell } 1319 \\
\text { (L403936) }\end{array}$ & $\begin{array}{l}11^{\mathrm{h}} 34^{\mathrm{m}} 13.2^{\mathrm{s}} \\
+40^{\mathrm{d}} 02^{\mathrm{m}} 36^{\mathrm{s}}\end{array}$ & 0.2906 & 4.395 & $\begin{array}{c}4.79_{-0.49}^{+0.51} \\
\text { Intermediate }\end{array}$ & $\begin{array}{l}0.013 \\
0.148^{b}\end{array}$ & 23.80 & 351 & 135 \\
\hline $\begin{array}{c}\text { RXC J1501.3+4220 } \\
- \text { Z7215 - } \\
\text { (L371804) }\end{array}$ & $\begin{array}{l}15^{\mathrm{h}} 01^{\mathrm{m}} 23.0 \mathrm{~s} \\
+42^{\mathrm{d}} 20^{\mathrm{m}} 40^{\mathrm{s}}\end{array}$ & 0.2917 & 4.406 & $\begin{array}{c}5.87_{-0.41}^{+0.42} \\
\text { Intermediate }\end{array}$ & $\begin{array}{c}0.096 \pm 0.06 \\
0.052 \pm 0.09\end{array}$ & 24.13 & 422 & 162 \\
\hline
\end{tabular}

Notes. The mass listed for Abell 1319 and Z7215 are SZ mass-estimates from Planck (Planck Collaboration XXIX 2014). The last three columns give the characteristics of a radio halo falling on the $P-M$ correlation from Cassano et al. (2013) for a cluster corresponding to that mass. We use these correlation values to compare to the diffuse emission detected by LOFAR. ${ }^{(a)}$ Our mass-estimate from XMM-Newton observations and scaling relations from Reichert et al. (2011). Since this cluster is at such a low mass, the $P-M$ correlation is not applicable. ${ }^{(b)} w$ and $c$ parameters are computed for Abell 1319-A only.

our LOFAR image, no diffuse sources appear to be present at the ICM centres nor on the ICM edges. We do not estimate upper limits for diffuse emission at the centre of each ICM component because residual AGN emission from the BCGs is too prominent for a reliable subtraction. We also cannot compare the residual emission to the $P-M$ correlation since we do not have the individual mass estimates for each clump, and each clump likely has a mass lower than cluster masses tested in the $P-M$ correlation.

Since the active BCGs all appear to reside in their respective ICM centres, and since no radio haloes or radio relics are detected in this system, we suggest that this multi-component system is in a pre-merging state, i.e. the separate ICM components are in gravitational infall, but have not yet merged. The centroid shift and the concentration parameter for Abell 1319-A, the eastern clump, also indicate that this ICM component is more relaxed, according to the boundaries on the $w-c$ diagram (with $w=0.013$, and $c=0.148$ from Fig. 1; also see Cassano et al. 2010, 2016; Cuciti et al. 2015). These parameters are measured from our Chandra X-ray image of Abell 1319.

\subsection{Abell 1314: Example of a merging system}

Abell 1314 has been studied for several decades with radio telescopes, starting with Webber (1974). There are four IC galaxies in the cluster with radio counterparts. Two BTs, associated with IC 708 and IC 711, were identified in 1976 with the Westerbork telescope at $610 \mathrm{MHz}$ and $5 \mathrm{GHz}$ (Vallee \& Wilson 1976). IC 708 exhibits a wide-angle opening between two tails, both with short extents and radio lobes to the west of the host. IC 711 is a head-tail, or narrow-angle tail, with a single, long radio tail extending hundreds of kpc north of the host galaxy.

Srivastava \& Singal (2016) presented GMRT observations at 235, 610, and $1300 \mathrm{MHz}$ of the head-tail IC 711 in Abell 1314. These authors produced a spectral index map over the body of the emission and report a break in the spectrum. They suggest that the morphology of the tail did not form solely from $\mathrm{N}-\mathrm{S}$ movement from the host galaxy because there is a sharp turn in the emission at the northernmost extent. More recently, Sebastian et al. (2017) presented a low-resolution image from a $240 \mathrm{MHz}$ GMRT observation of the radio tail associated with IC 711. In this image, it is apparent that there is more emission extending westward at the northernmost region referred to by Srivastava \& Singal (2016).

An overlay of our LOFAR images of Abell 1314, in high and low resolution, can be seen in Fig. 4. The LOFAR highresolution $(8 \operatorname{arcsec} \times 5$ arcsec $)$ image of Abell 1314 reveals extended tail emission from IC 711. The wide-angle tail IC 708 is also visible to the west of the elongated head-tail. The sharp turn noted by Srivastava \& Singal (2016) actually appears to be filamentary emission sweeping westward for $\sim 300 \mathrm{kpc}$. As noted by Srivastava \& Singal (2016), this northernmost emission that appears, in projection, perpendicular to the length of the tail likely did not form from the trajectory of the active host galaxy. It is more likely that the radio emission in the northern part of the tail has been disturbed by the ICM, such as ram pressure from turbulence or shocks traveling from the cluster centre outward and toward the west. The LOFAR low-resolution map (shown as red contours and colour in Fig. 4) reveals an additional $300 \mathrm{kpc}$ of bulk diffuse emission beyond this high-resolution filamentary portion of the tail, giving a total projected size of $800 \mathrm{kpc}$.

In our low-resolution LOFAR image, faint diffuse emission is also detected in the central regions of the cluster, which has not been detected at higher frequencies. This emission is not spherically uniform in shape or brightness, but instead exhibits "arms". It is possible that this emission is remnant AGN emission from the central BCG (IC 712: $z=0.033553$, which shows a compact core) or from other radio galaxies within the cluster centre.

\subsubsection{Spectral index on radio emission}

A spectral index map is generated along the head-tail radio galaxy IC 711 by comparing the GMRT map at $610 \mathrm{MHz}$ to our LOFAR map at $144 \mathrm{MHz}$. The spectral index along the tail steepens from south to north, but there is a region of flattening that takes place in the central part of the tail. The index goes from $\alpha \sim-2$ to -1.3 and then back to $\sim-2$ within this region. A compact source is also seen to the northeast edge of the tail emission, marked by a flat spectral index, but this is likely to be a foreground source. The spectral index in the northernmost portion of the tail, where emission sweeps westward, cannot be determined since this emission is not detected by the archival GMRT observations we obtained at 610 nor $235 \mathrm{MHz}$. 


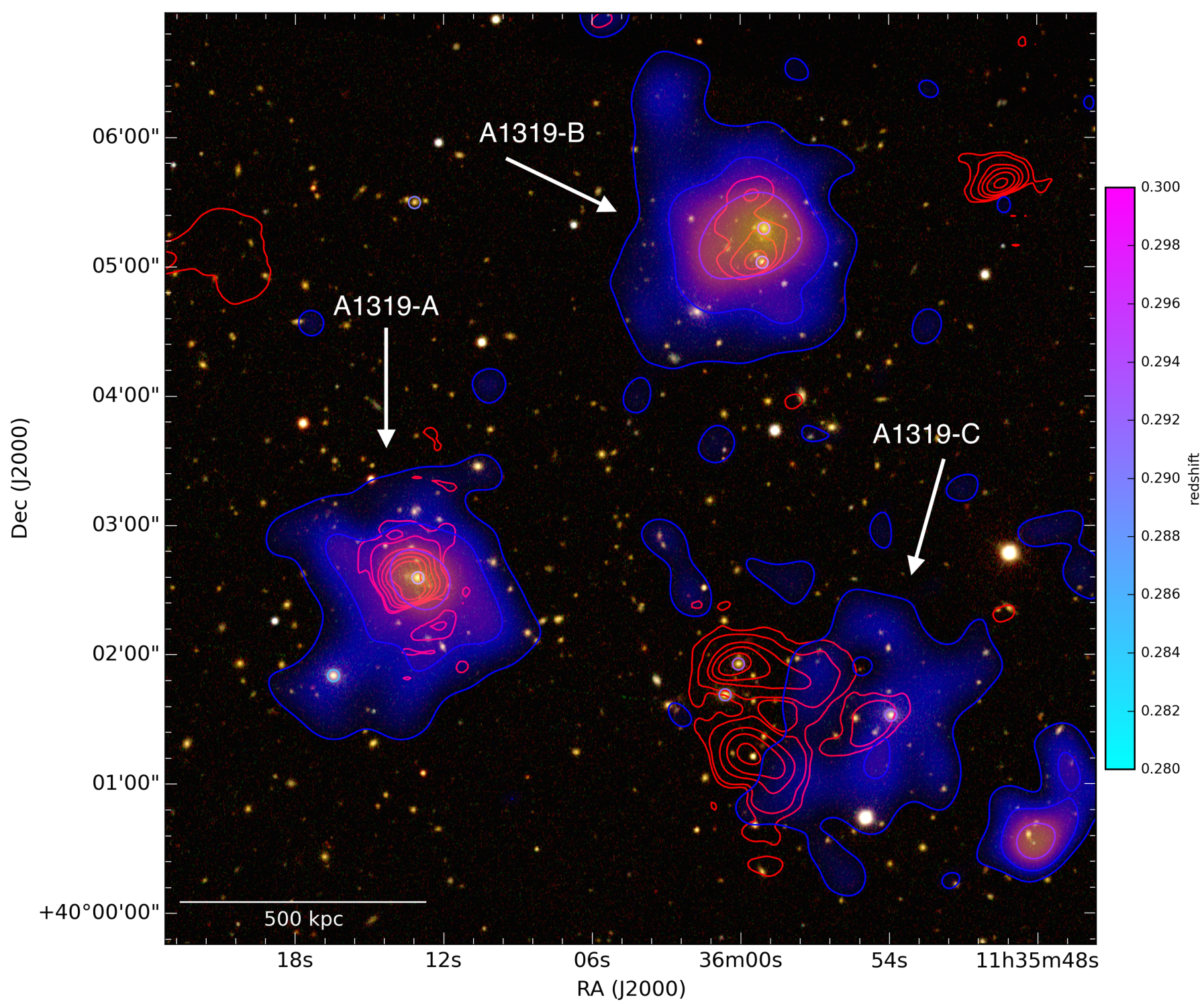

Fig. 2. SDSS $i, r, g$ image of Abell 1319 with LOFAR radio emission overlaid as contours. LOFAR high-resolution emission $(11$ arcsec $\times 6$ arcsec $)$ is shown by red contours $[3,5,16,64,128,256,512] \times \sigma$ where $\sigma=170 \mu \mathrm{Jy}_{\text {beam }}^{-1}$. The smoothed Chandra X-ray image is shown in bluemagenta and as blue contours. Cluster galaxies with known spectroscopic redshifts are indicated by coloured rings, where the colour represents redshift.

To determine an upper limit on the spectral index of the diffuse emission at the cluster centre, we compare the flux density within $3 \sigma$ contours in our $144 \mathrm{MHz}$ LOFAR map to the same region of the $610 \mathrm{MHz}$ GMRT map ${ }^{6}$. This flux density within this region is only at noise level at $610 \mathrm{MHz}$, so we integrate the noise $\left(300 \mu \mathrm{Jy} \mathrm{beam}^{-1}\right)$ over the area of the $3 \sigma$ contour region from the LOFAR map and find the upper limit on the flux density to be $19.4 \mathrm{mJy}$ at $610 \mathrm{MHz}$. Comparing the measurements gives a spectral index upper limit estimate of $\alpha<-1.3$.

\subsubsection{Central diffuse radio emission}

The non-spherical, limb-like emission at the centre of Abell 1314 , with a largest linear size of $\sim 380 \mathrm{kpc}$, is probably not related to a cluster radio halo. The flux density of the central diffuse emission is measured in our compact-source-subtracted

\footnotetext{
6 We compare the images made with the same clean settings including uniform weighting and the same beam size.
}

image made with a $u v$-taper of 30 arcsec. The flux density within $3 \sigma$ contours where $\sigma=400 \mu \mathrm{Jy}$ beam $^{-1}$ is $109 \pm 11 \mathrm{mJy}$. This translates to a power of $(2.85 \pm 0.29) \times 10^{23} \mathrm{~W} \mathrm{~Hz}^{-1}$ at $144 \mathrm{MHz}$ and $(1.48 \pm 0.15) \times 10^{22} \mathrm{~W} \mathrm{~Hz}^{-1}$ at $1.4 \mathrm{GHz}$ if extrapolated assuming a spectral index of $\alpha=-1.3$. This power of $\log _{10}\left(\mathrm{P}_{1.4} / \mathrm{W} \mathrm{Hz}^{-1}\right)=22.17$ is much lower than the power of radio haloes even at the lowest end of the cluster mass range (see the $P-M$ correlation from Cassano et al. 2013 and Martinez Aviles et al. 2016). As we discuss in Sect. 4.2, it is more plausible that this emission is a remnant radio galaxy.

\subsubsection{X-ray results on Abell 1314}

Figure 6 shows the $0.3-2.0 \mathrm{keV} \mathrm{X}$-ray band image of Abell 1314 obtained with XMM-Newton together with LOFAR highresolution radio contours. As is clear from Fig. 6, Abell 1314 shows an elongated and disturbed morphology indicating that it is still at a dynamically young state. 


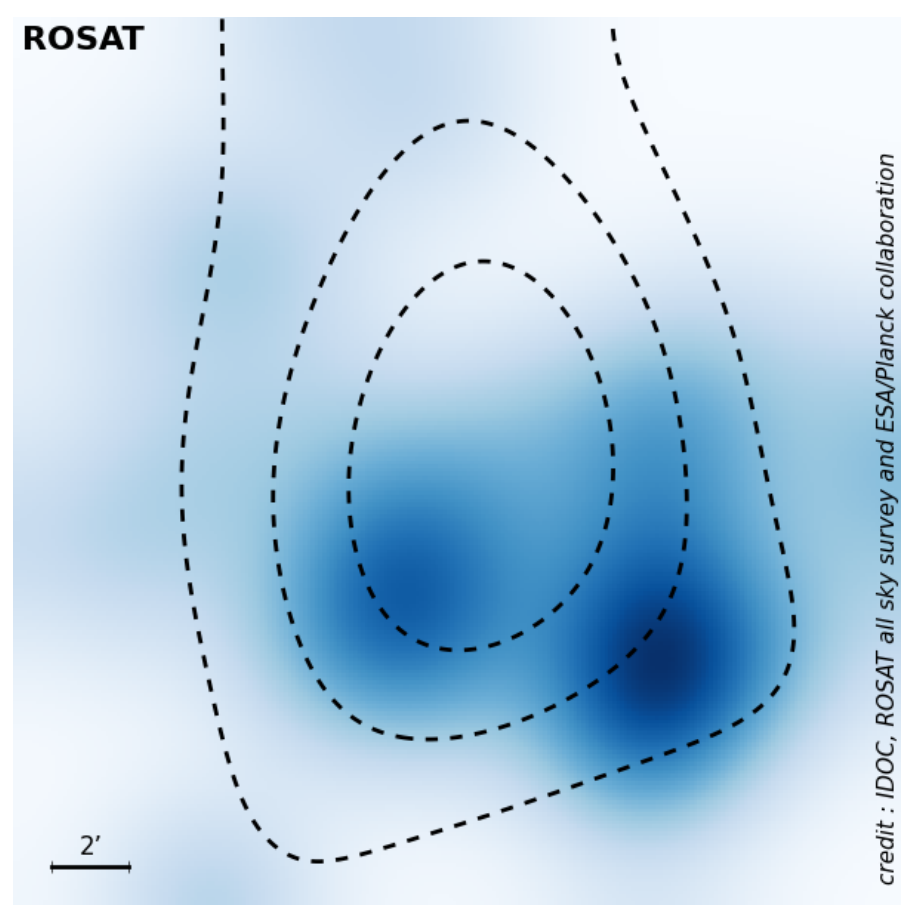

Fig. 3. Image taken from the Planck SZ Cluster Database. Abell 1319-A is accompanied by two additional cluster components of similar size at a similar redshift. We suggest the SZ-derived mass is a combination of three low-mass cluster components (faintly visible in blue, as seen by ROSAT).

Using XMM-Newton MOS data, we characterised the dynamical state of cluster and investigate the relationship between thermal (ICM) and non-thermal (radio) components. We derived the morphological parameters $\left(c_{100 \mathrm{kpc}}\right.$ and $\left.w_{500 \mathrm{kpc}}\right)$ as $0.063 \pm 0.003,0.026 \pm 0.01$, respectively. The morphological parameters of Abell 1314 are in good agreement with those observed for merging clusters (see Fig. 1); however, we note that the values for Abell 1314 are computed from XMM data whereas the values of other clusters we use for comparison are computed from Chandra data. XMM has a larger point spread function (PSF), and this is taken into account in the errors on $w$ and $c$.

For the surface brightness profile, we used $0.3-2.0 \mathrm{keV}$ energy range and fitted it with the PROFFIT software package (Eckert et al. 2011). We extracted the surface brightness profile in the western sector with an opening angle of $40^{\circ}$ (covering the full region where the spectral index is seen to flatten). The resulting surface brightness profile is shown in the Fig. 7. The discontinuity in XMM-Newton surface brightness profile is clearly visible. The location of the discontinuity around $r \sim 10^{\prime}$, is just outside of the BCG, IC 712. In order to characterise the discontinuity, we assume that the gas density follows two powerlaw profiles connecting at a discontinuity with a density jump. The density profile was projected onto the line of sight with the assumption of spherical symmetry. All the parameters of the model were let free in the fit. The surface brightness profile was well fitted with the above model (reduced $\chi^{2}=1.16$ for $32^{\circ}$ of freedom). The best-fitting broken power-law model is shown in Fig. 7. The compression factor and the location of the discontinuity are $C=2.1 \pm 0.2$ and $r=10.8 \pm 0.3 \mathrm{arcmin}$, respectively.

Since the discontinuity is located near the edge of the field of view of the MOS instrument, the determination of the instrumental background might be inaccurate. We investigate the impact of this systematic effect by changing the normalisation of the instrumental background $\pm 20 \%{ }^{7}$. The effect of the systematic error on the location of the discontinuity and the compression factor are smaller than or compatible with the statistical errors. In the following discussion section, we used the error defined by $\sigma \equiv \sqrt{\sigma_{\text {stat }}^{2}+\sigma_{\text {syst }}^{2}}$, which leads $C=2.1 \pm 0.4$ and $r=10.8 \pm 0.4$ arcmin, respectively.

Since Abell 1314 is not detected by Planck, we must estimate its mass from X-ray data. We estimate the mass of Abell 1314 from the spectrum of the XMM-Newton observation. The $0.5-2.4 \mathrm{keV}$ band flux of Abell 1314 is $F_{x}=6.13 \times$ $10^{-12} \mathrm{erg} \mathrm{s}^{-1} \mathrm{~cm}^{2}$. The luminosity distance of Abell $1314(z \sim$ 0.034 ) is $150.3 \mathrm{Mpc}$ therefore, the estimated X-ray luminosity is $L_{x}=1.7 \times 10^{43} \mathrm{erg} \mathrm{s}^{-1}$ and the estimated ICM temperature is $k T \sim 1.7 \mathrm{keV}$. Our result is in good agreement with the $L_{x}-T$ relation from Xue \& Wu (2000; Fig. 1 in their paper). With this temperature we estimate the mass with the scaling relation in Reichert et al. (2011), which is based on 14 literature samples, and find $M_{500} \sim 0.68 \times 10^{14} M_{\odot}$. Our estimated mass is broadly consistent with that from $\operatorname{MCXC}\left(M_{500}=0.4608 \times 10^{14} M_{\odot}\right.$; Piffaretti et al. 2011) ${ }^{8}$.

\subsection{Z7215: Example of post-merging system}

An overlay of our LOFAR images of the cluster Z7215, in high and low resolution, can be seen in Fig. 8. This overlay includes smoothed X-ray contours from Chandra data. According to the Chandra map, the thermal ICM appears to be moderately disturbed and elongated. The cluster does not host a cool core and looks moderately asymmetric. Because of the low counts and the position of the chips we cannot give reasonable estimates of $w$ and $c$ from the Chandra data alone. An XMM-Newton observation of Z7215 is used instead to estimate values for the centroid shift and concentration parameters, as was done for Abell 1314, but these estimates have larger errors: $w=0.096 \pm 0.06$ and $c=0.052 \pm 0.09$. These values and the estimated $\beta$ parameter of $0.84 \pm 0.07$ indicate a disturbed morphology. The estimated core radius $R_{\mathrm{c}}$ is $1.29 \pm 0.10 \mathrm{arcmin}$. Considering the distance to this cluster $(1 \operatorname{arcmin} \sim 260 \mathrm{kpc}$ ), the core radius is $\sim 350 \mathrm{kpc}$, which is apparently on the larger side (e.g. Mohr et al. 1999).

The LOFAR image of Z7215 at high resolution does not show many radio sources associated with or located at the cluster centre. A FR-II radio galaxy is seen north of the cluster, coincident with an X-ray source seen by Chandra, but this source is likely in the background since it overlaps galaxies at higher redshifts. After subtracting sources and re-imaging at a lower resolution, diffuse emission is visible above $3 \sigma$ at the cluster centre, where $\sigma=450 \mu \mathrm{Jy}_{\text {beam }}{ }^{-1}$. This diffuse emission is coincident with the Chandra X-ray contours at the cluster centre. However, this diffuse emission is not spherical in morphology, and instead appears to be elongated to the west, with a minor axis about half the size of the major axis (about $500 \mathrm{kpc}$ in the N-S direction and $1 \mathrm{Mpc}$ in the $\mathrm{E}-\mathrm{W}$ direction). There is also a portion of diffuse emission detached from the centre on the $\mathrm{N}-\mathrm{W}$ edge, but it overlaps several potentially active galaxies, coincides with an $\mathrm{X}$-ray source seen by Chandra, and is also more compact in its

\footnotetext{
7 We use a conservative, and likely overestimated, error value of $20 \%$ and note that the results and conclusion do not change with or without the systematic error.

8 There is a $\sim 30 \%$ difference between our mass estimate and the mass estimate from Piffaretti et al. (2011), which is within the scatter of the $L_{x}-M$ scaling relationship.
} 


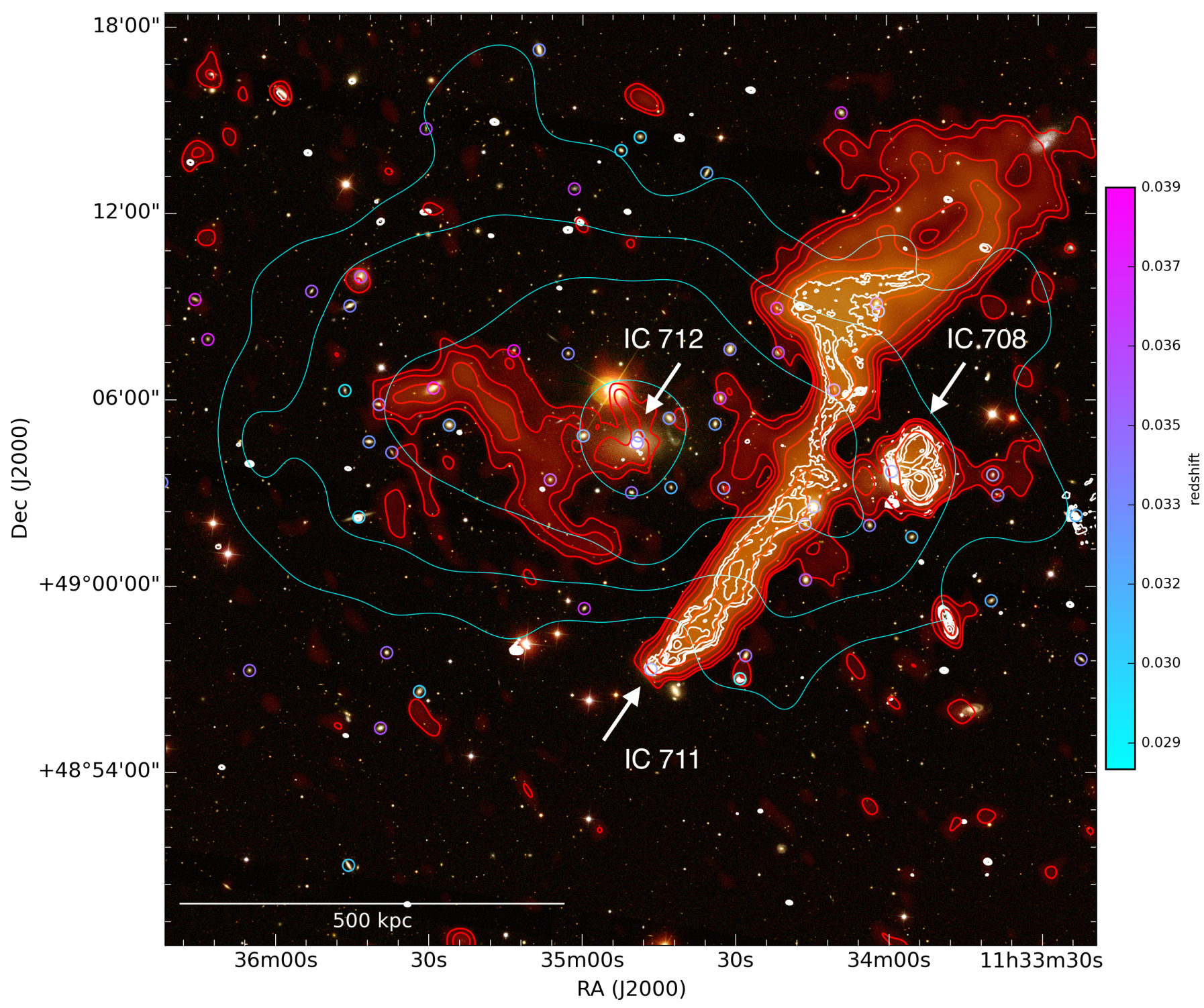

Fig. 4. SDSS $i, r, g$ image of Abell 1314 with radio emission overlaid as contours. LOFAR high-resolution ( 8 arcsec $\times 5$ arcsec) emission is shown by white contours $[6,12,24,48,96,192,384,768] \times \sigma$ where $\sigma=180 \mu \mathrm{Jy}_{\text {beam }}{ }^{-1}$. LOFAR diffuse emission after compact source subtraction (with a resolution of $43 \operatorname{arcsec} \times 33$ arcsec) is shown in red and red contours where contours are $[3,6,12,24,48] \times \sigma$ and $\sigma=300 \mu \mathrm{Jy}$ beam ${ }^{-1}$. Smoothed XMM-Newton X-ray contours as also shown in cyan. Cluster galaxies with known spectroscopic redshifts are shown by coloured rings, where the colour represents redshift.

extent. We do not include this emission on the northwest edge, which is likely of AGN origin, as part of the central diffuse emission when measuring flux densities and calculating powers in the following subsections.

We discover centrally located diffuse emission in Z7215, which is most likely a radio halo, although it is not spherically uniform in its shape and it is dimmer than expected from the $P-M$ correlation. The flux density of the central diffuse emission is measured in our compact-source-subtracted image made with a $u v$-taper of $30 \operatorname{arcsec}$ (resolution of $43 \operatorname{arcsec} \times 33 \operatorname{arcsec}$ ). The flux density above $2 \sigma$ contours where $\sigma=450 \mu \mathrm{Jy} \mathrm{beam}^{-1}$ is $20.2 \pm 2.0 \mathrm{mJy}$. This translates to a power of $(5.91 \pm 0.60) \times$ $10^{24} \mathrm{~W} \mathrm{~Hz}^{-1}$ at $144 \mathrm{MHz}$ and $(3.07 \pm 0.31) \times 10^{23} \mathrm{~W} \mathrm{~Hz}^{-1}$ at $1.4 \mathrm{GHz}$ if extrapolated assuming a spectral index of $\alpha=-1.3$. This power of $\log _{10}\left(\mathrm{P}_{1.4} / \mathrm{W} \mathrm{Hz}^{-1}\right)=23.49$ is slightly less than the expected power of a halo fitting on the correlation at $1.4 \mathrm{GHz}$ (see Table 1), but close to the powers of other radio haloes at this cluster mass (see Fig. 9). A $610 \mathrm{MHz}$ observation covering
Z7215, as part of the GMRT Radio Halo Survey, was analysed by Venturi et al. (2008), and they found an upper limit for diffuse radio emission at the cluster centre: $\log _{10}\left(\mathrm{P}_{610} / \mathrm{W} \mathrm{Hz}^{-1}\right)=$ 24.20. Extrapolating this value to $1.4 \mathrm{GHz}$ with a spectral index of $\alpha=-1.3$ gives $\log _{10}\left(\mathrm{P}_{610} / \mathrm{W} \mathrm{Hz}^{-1}\right)=23.73$. This value is comparable but slightly larger than the power we extrapolate from our $144 \mathrm{MHz}$ LOFAR observations.

To test the capability of our LOFAR observation to recover such faint extended emission, we inject a modeled radio halo with a radius $R_{\mathrm{H}}$ determined by the correlations from Cassano et al. (2013; $R_{\mathrm{H}}=422 \mathrm{kpc}$ and $r_{e}=162 \mathrm{kpc}$ for a cluster with $\left.M_{500}=5.87 \times 10^{14} M_{\odot}\right)$. We inject the mock radio halo in a relatively empty region of the cluster field (at RA, Dec: $\left.15^{\mathrm{h}} 03^{\mathrm{m}} 04.3^{\mathrm{s}},+42^{\mathrm{d}} 34^{\mathrm{m}} 49.4^{\mathrm{s}}\right)$. The mock halo is initially assumed to have a power and central brightness, $I_{0}$, defined by the correlation, and we lower the injected flux until the recovered mock halo emission is comparable to the detected emission at the cluster centre. We measure and compare the recovered 


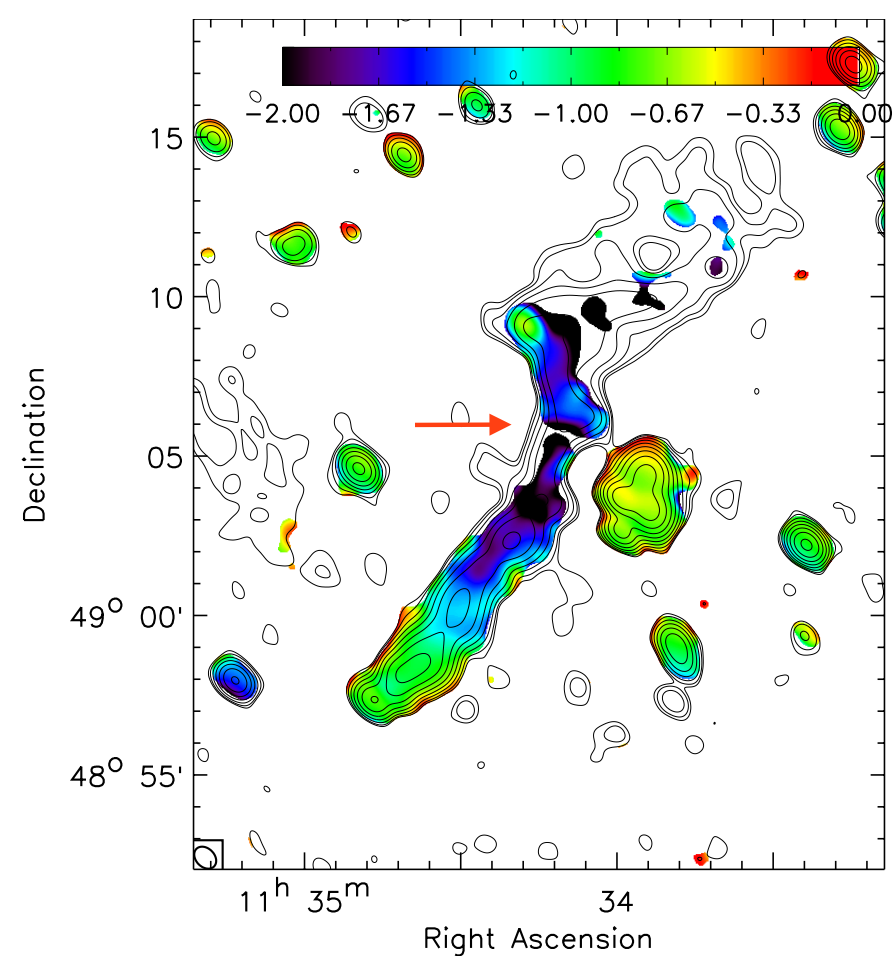

Fig. 5. Spectral index map over the BT galaxies in Abell 1314. The map is made with the LOFAR map at $144 \mathrm{MHz}$ and the GMRT map at $610 \mathrm{MHz}$. Both maps are imaged with the same setting and convolved to the same beam size. We indicated no RA, Dec offset between the two maps. The radio head-tail from IC 711 shows steepening in the length of the tail with a portion of flattening in the mid-section. A red arrow roughly marks where the flattening of the spectral index occurs.

flux density of the injected source to the flux density of the diffuse emission at the cluster centre above $2 \sigma$ and determine at which injected flux value they are approximately equal. The flux density of the recovered halo is measured in our LOFAR low-resolution image ${ }^{9}$ above $2 \sigma$ contours and is found to be $19.3 \pm 1.9 \mathrm{mJy}$. This recovered flux density of the mock halo is about equal, within error, to the central diffuse source. The injected flux value of this halo is $26.3 \mathrm{mJy}$, and therefore the recovered flux is $\sim 73 \%$ of the injected flux. These findings demonstrate that our LOFAR observation is able to partially, but not fully, recover the faint extended emission of this radio halo. Therefore, we conclude that the true flux density of the radio halo in Z7215 is more reasonably approximated by the injected flux density value of the mock halo. We extrapolate this flux density to $1.4 \mathrm{GHz}$ assuming a spectral index of $\alpha=-1.3$ and find a power of $3.99 \times 10^{23} \mathrm{~W} \mathrm{~Hz}^{-1}$. We compare this power to the power of the detected emission above $2 \sigma$ and to the powers of a sample of radio haloes, as shown in Fig. 9.

\section{Discussion}

\subsection{Abell 1319}

The three ICM clumps, A, B, and C, are separated by a distance of about $\sim 500 \mathrm{kpc}$. It is not likely that this system is post-merger, as we might then expect to see X-ray filaments or an overall elongated, disturbed ICM of the combined components. However, a deeper X-ray observation would be needed to exclude the

\footnotetext{
9 Made with a $u v$-taper of 30 arcsec and Briggs' robust parameter of 0 .
}

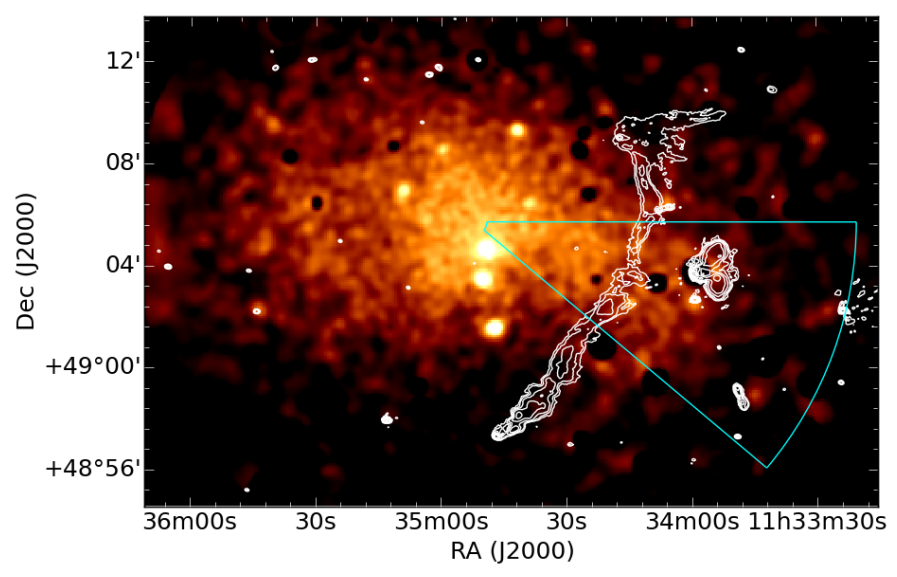

Fig. 6. XMM-Newton map of Abell 1314 with LOFAR high resolution overlaid as white contours $[6,12,24,48,96,192,384,768] \times \sigma$ where $\sigma=180 \mu \mathrm{Jy} \mathrm{beam}^{-1}$. The cyan region indicates where the surface brightness is analysed along the length of the tail, starting from the cluster centre and going to a radius of $600 \mathrm{kpc}$.

presence of such filaments. Additionally, if this system was postmerger the ICM of Abell 1319-A would be expected to be more disturbed with a higher $w$ value and a lower $c$ value.

Diffuse cluster-scale sources do not appear in our LOFAR images. We suggest that there are no radio relics or radio haloes associated with this multi-component system. The lack of ICM radio sources lends further support to the hypothesis that this system is in its pre-merging phase since radio relics and radio haloes are reliable tracers of mergers.

\subsection{Abell 1314}

Although there is a central diffuse source in this cluster, its morphology, scale, and brightness distribution is unlike that of a cluster radio halo. The power of the central diffuse source lies an order of magnitude below the $P-M$ correlation, even at the low end of the mass range, suggesting that this source is not similar to a radio halo. Indeed, this cluster is considered to have a very low mass, and the $P-M$ correlation has not yet been tested for such low-mass merging clusters. Although Abell 1314 is highly disturbed (see Fig. 6 and $w, c$ parameters in Table 1), it is not so surprising that a halo is not detectable given the mass estimate we derive. It is possible that the ICM has been re-energised by merger turbulence, but a spherical, cluster-wide halo has not been generated.

One may speculate whether this elongated patch of radio emission is the beginning, or making, of a radio halo, that will develop into a full-scale halo at a later stage. Donnert et al. (2013) used simulations to show that radio haloes are transient sources with an evolving spectrum, and identified three stages of radio/X-ray activity: infall, re-acceleration, and decay. It is unlikely that Abell 1314 is in the re-acceleration phase where a cluster-scale, spherically uniform halo is expected to form. Our deep observations should have revealed such a halo, however, we only detect faint arms of emission at the centre of Abell 1314.

The central diffuse source, which likely has a steep spectrum since it is not detected by GMRT observations at 235 or $610 \mathrm{MHz}$, (an estimate of the spectral index in Sect. 3.2.1 gives $\alpha \sim-1.3$ ) is similar to the irregular large-scale emission recently discovered in Abell 1931 by Brüggen et al. (2018). In that paper, they report a remnant radio galaxy that is detected by LOFAR at $144 \mathrm{MHz}$. Another $144 \mathrm{MHz}$ LOFAR observation 

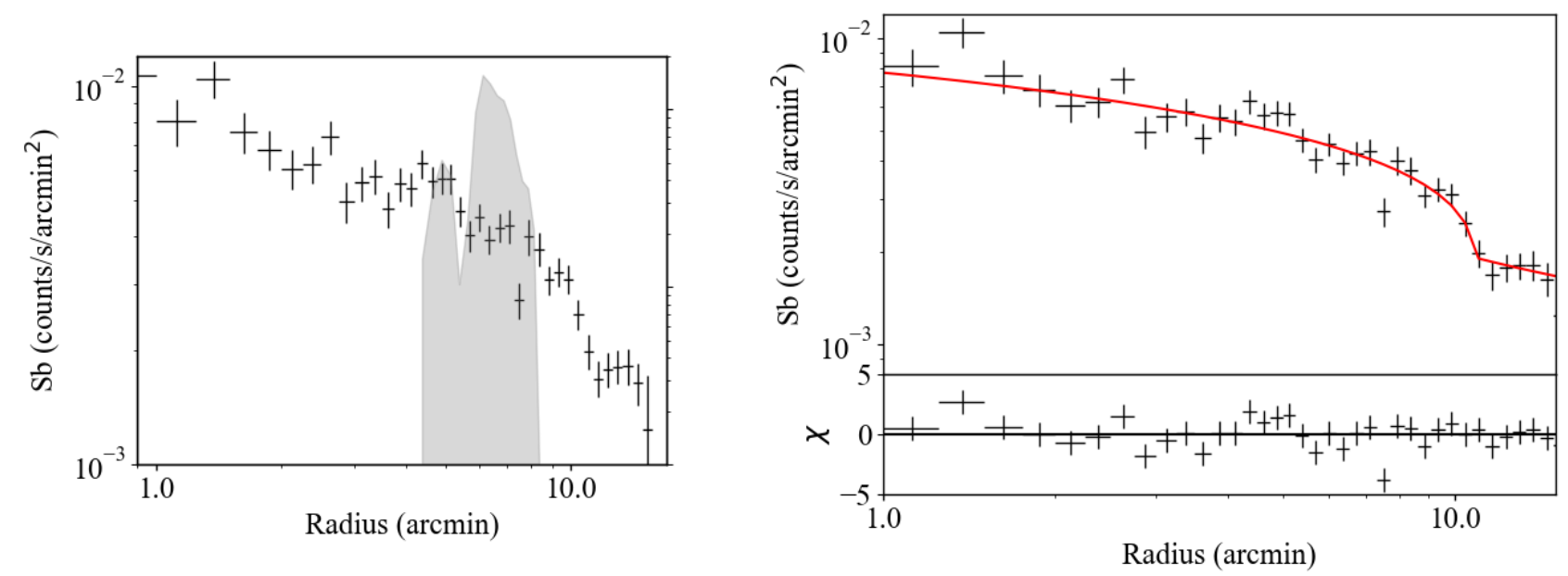

Fig. 7. Left panel: XMM-Newton $0.3-2.0 \mathrm{keV}$ band surface brightness profile. The grey shaded area represents the LOFAR radio brightness profile (in arbitrary units). Right panel: same as left but with the best-fitting broken power-law model.

of the galaxy group MaxBCG J199.31832+51.72503 showed an extended radio galaxy embedded in steep, remnant radio emission at the group's centre (Savini et al. 2018). We may be witnessing a similar phenomena in Abell 1314 where the arms of emission are old, fading remnant lobes of a previous activity cycle from the central BCG AGN. However, there are multiple galaxies at the cluster redshift that reside within this diffuse radio source, hence the radio emission could be a superposition of multiple remnant lobes. A question remains as to whether this diffuse source is simply fading AGN emission, or if this fossil emission has been partially re-accelerated, and therefore slightly re-brightened, by merger turbulence. Without detailed spectral index maps this will be impossible to untangle.

Still, this is yet another example that LOFAR is able to detect old, faded, and faint large-scale radio sources. This fossil radio emission proves that relativistic electrons are filling the inner part of the cluster volume. As LOFAR reveals more and more fossil radio emission from remnant AGN, we may begin to see a clear connection between these sources and ICM clusterscale sources such as radio haloes (e.g. Wilber et al. 2018a; Brüggen et al. 2018).

\section{IC 711}

The extended radio emission produced by the galaxy IC 711 shows a spectral index that generally steepens from the head to the end of the tail (see Fig. 5), as is expected of typical headtail radio galaxies. However, there is a portion of the tail, near the middle, that shows signs of flattening. This flattening likely occurs because the emission in this region is disturbed or compressed. We suggest that the disturbance induces re-acceleration, which causes the electrons to emit at slightly higher energies in this region. In fact, the flattening occurs where there is a concentration of thermal ICM emission, in the same plane of the cluster merger. This flattening is similar to the gentle reenergisation (GReET) phenomena reported by de Gasperin et al. (2017), where the spectral index of a wide-angle-tail (WAT) source is seen to increase where it is expected to decrease.

A small jump can be seen at the location of the radio tail of IC 711 from a radial X-ray surface brightness profile of the cluster. A discontinuity is also seen in the eastward direction (not shown), but there are no significant radio sources on this side of the cluster. It is possible that bulk motions in the ICM distort the tail, which flows perpendicular to the merger direction. The total shape of the tail has a slight arc that is concave with respect to the cluster centre. In the high-resolution image, we see thin filamentary structures in the northern emission of the tail, which appear to be sweeping from east to west. This filamentary emission is even more suggestive of disturbance, such that this sweeping effect may have been produced by turbulence and shocks traveling from the cluster centre to the outskirts. It is impossible to tell whether this northernmost emission has been re-accelerated, or compressed by a shock because there is no radio emission in this region present in our GMRT images and the XMM-Newton map is not sensitive enough to detect a jump in temperature or surface brightness in this region. The $240 \mathrm{MHz}$ GMRT image produced by Sebastian et al. (2017) may be helpful to estimate a spectral index in this region, but such an estimate could be unreliable since their observing frequency is close to our LOFAR observing frequency.

\section{3. $Z 7215$}

The power of the diffuse source at the cluster centre is below the $P-M$ correlation, but comparable to the powers of other radio haloes at a similar cluster mass (see Fig. 9). It is likely that this diffuse source, elongated in the E-W direction and coincident with the thermal $\mathrm{X}$-ray emission of the cluster, is a radio halo. There are no compact sources seen in our high-resolution LOFAR image that reside within the cluster centre, indicating it is unlikely that the diffuse emission is of AGN origin. The flux density of the central diffuse source in $\mathrm{Z} 7215$ (contained within $2 \sigma$ contours) is roughly equal to the flux density of the emission recovered after injecting a fake radio halo with a flux density of $26.3 \mathrm{mJy}$ at $144 \mathrm{MHz}$.

Based on our findings, the radio halo in Z7215 is underluminous. One reason for this could be that the halo is smaller than typical haloes fitting the correlation. Indeed the halo as seen by LOFAR appears shortened in the $\mathrm{N}-\mathrm{S}$ direction, and we measure an effective radius $R_{\mathrm{H}}=\sqrt{500 \mathrm{kpc} \times 250 \mathrm{kpc}} \approx$ $350 \mathrm{kpc}$. Another possibility is that the halo is an USSRH. These sources are predicted (Cassano et al. 2010; Donnert et al. 2013) and observed to be typically under-luminous with respect to the correlation (see Fig. 9); USSRHs are thought to form in less massive and less energetic merger events or at the beginning or fading evolutionary phase of halo formation (Cassano et al. 2006; 


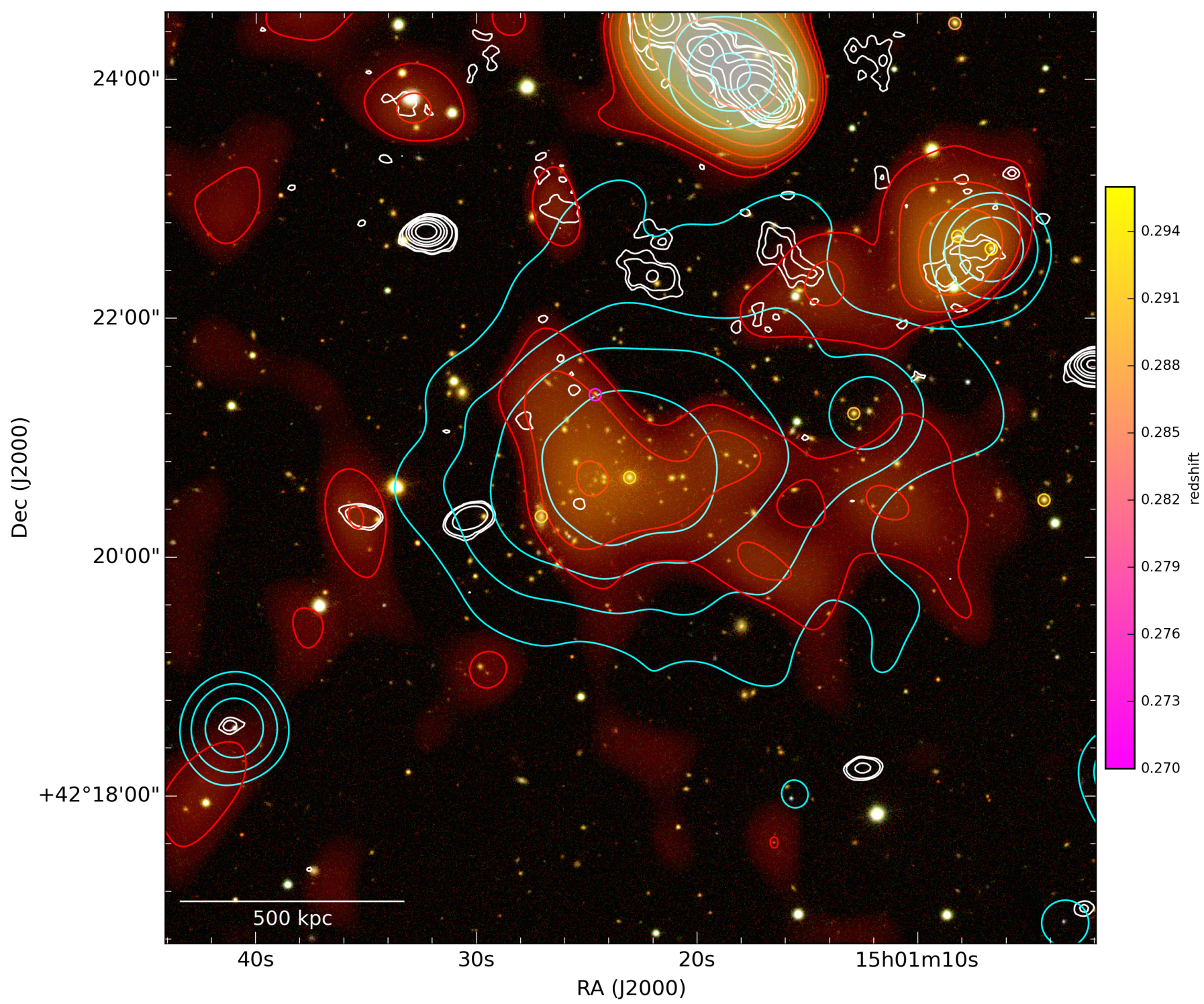

Fig. 8. SDSS $i, r, g$ image of Z7215 with LOFAR radio emission overlaid as contours. The LOFAR high-resolution $(10$ arcsec $\times 6$ arcsec $)$ emission is shown by white contours $[3,5,16,64,128,256,512] \times \sigma$ where $\sigma=170 \mu \mathrm{Jy}^{\text {beam }}{ }^{-1}$. The LOFAR diffuse emission after compact source subtraction (with a resolution of $40 \operatorname{arcsec} \times 34 \mathrm{arcsec}$ ) is shown in red colour and red contours where contours are $[2,4,8,16,32,64] \times \sigma$ and $\sigma=350 \mu \mathrm{Jy} \mathrm{beam}^{-1}$. Smoothed Chandra X-ray contours are also shown in cyan. Cluster galaxies with known spectroscopic redshifts are marked by coloured rings, where the colour represents redshift.

Brunetti et al. 2008; Donnert et al. 2013). Since the actual halo radius is smaller than the radius assumed for the upper limit computed by Venturi et al. (2008), the upper limit should be smaller and the resulting spectral index should be steeper. Deeper observations around $330 \mathrm{MHz}$ could help to constrain the spectral index.

This cluster was also on the edge of our LoTSS observation pointing, which may have affected the amount of emission that was detected and recovered. Indeed, only $73 \%$ of the injected halo flux was recovered in our LOFAR image. Follow-up observations centred on this cluster are already planned and scheduled with LOFAR. Observations centred on the cluster may reveal more flux associated with the radio halo. Future LOFAR low band antenna observations may also be of interest in conjunction with the HBA observations so that the spectral index could be constrained. In conclusion we claim that this cluster is in its post-merging phase, where the system has begun to relax. The radio halo we discover is under-luminous with respect to the correlation.

\section{Conclusions}

In summary we find following results:

- We show that Abell 1319 is a multiple-component system and suggest that these three low-mass clusters, or galaxy groups, are about to merge. The LOFAR radio observations reveal active BCG galaxies at the centres of all three ICMs. There are no diffuse cluster-scale ICM radio sources present in this system, such as radio relics or radio haloes, which supports the notion that this system is pre-merging.

- Abell 1314 is a low-mass cluster that shows a highly disturbed thermal ICM. Irregularly shaped diffuse radio emission present at the cluster centre is likely to be remnant AGN lobe emission from previous activity cycles of the currently active BCG IC 712. We argue that this cluster is in a merging phase, although it is likely too low of mass to generate a detectable radio halo, even at a later evolutionary stage. The extended head-tail radio galaxy IC 711 shows signs of 


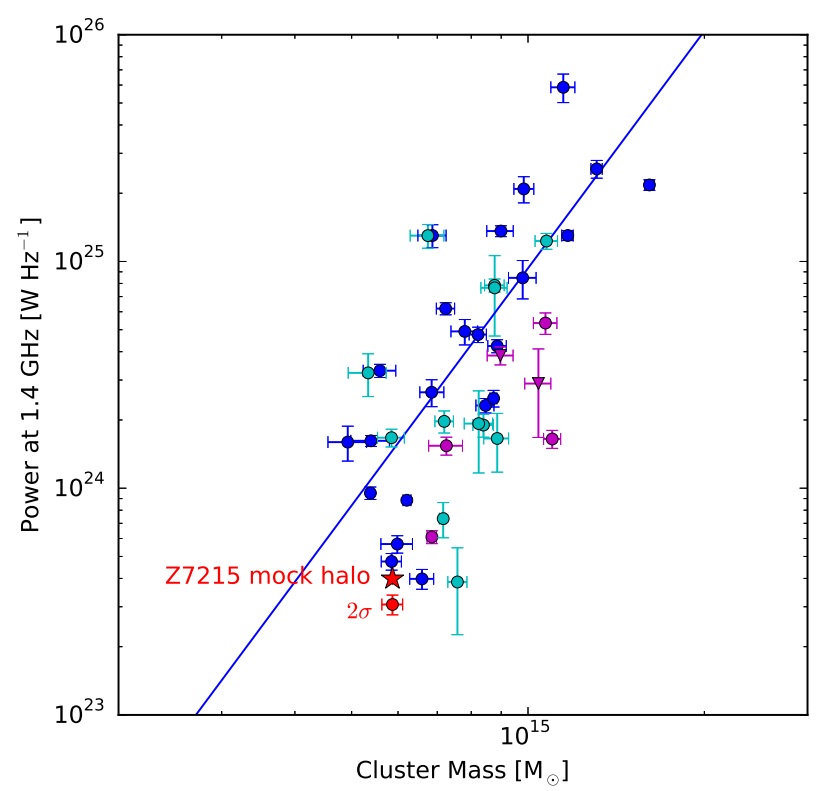

Fig. 9. Sample of radio haloes plotted by their radio power at $1.4 \mathrm{GHz}$ vs. their cluster mass $\left(M_{500}-\right.$ as determined from Planck observations). The sample of haloes and their correlation is reproduced from Martinez Aviles et al. (2016). Haloes with flux measured at $1.4 \mathrm{GHz}$ are indicated by blue circles and their derived fit is shown as a blue line. Cyan circles represent haloes with flux measured at frequencies other than $1.4 \mathrm{GHz}$. Magenta circles represent ultra-steep haloes, and magenta triangles represent ultra-steep haloes with flux measured at frequencies other than $1.4 \mathrm{GHz}$. The red point shows the power computed from the emission detected in our LOFAR observation for Z7215. The red star indicates the power of our mock halo with a higher injected flux value. All halo powers include a k-correction with an averaged spectral index of $\alpha=-1.3$, as in Martinez Aviles et al. (2016).

disturbance. A spectral index map reveals that the middle portion of the tail, where the X-ray brightness is highest along the tail, indicates possible local re-acceleration or compression. The northernmost part of the tail shows filamentary emission that appears to be sweeping westward, suggestive of disturbance.

- We discover a radio halo in the massive, merging cluster Z7215 and we argue that this cluster is in its postmerging phase. The halo has a non-spherical morphology with a major axis of about $1 \mathrm{Mpc}$ and a minor axis of about $500 \mathrm{Mpc}$. The halo is dimmer than expected by the halo $P-M$ correlation but comparable to the powers of other haloes at similar cluster masses. We suggest that this could be a smaller halo or an ultra-steep spectrum halo. Future followups will distinguish between these possibilities.

Using LOFAR observations to add more clusters to this sample, specifically adding more examples of clusters which are in various stages of merging, would help to strengthen our understanding of how merging mechanisms are related to ICM radio sources.

Acknowledgements. LOFAR, the Low Frequency Array designed and constructed by ASTRON, has facilities owned by various parties (each with their own funding sources), and that are collectively operated by the International LOFAR Telescope (ILT) foundation under a joint scientific policy. The LOFAR software and dedicated reduction packages on https://github.com/ apmechev/GRID_LRT were deployed on the e-infrastructure by the LOFAR e-infragroup, consisting of J. B. R. Oonk (ASTRON \& Leiden Observatory), A. P. Mechev (Leiden Observatory), and T. W. Shimwell (Leiden Observatory) with support from N. Danezi (SURFsara) and C. Schrijvers (SURFsara). The data used in work was in part processed on the Dutch national e-infrastructure with the support of SURF Cooperative through grant e-infra $160022 \& 160152$ We thank the staff of the GMRT who have made these observations possible. The GMRT is run by the National Centre for Radio Astrophysics of the Tata Institute of Fundamental Research. This research made use of the NASA/IPAC Extragalactic Database (NED), which is operated by the Jet Propulsion Laboratory, California Institute of Technology, under contract with the National Aeronautics and Space Administration. AB acknowledges support from the ERC-StG 714245 DRANOEL. RJvW and HJAR acknowledge support from the ERC Advanced Investigator program NewClusters 321271 and RJvW acknowledges the VIDI research program with project number 639.042 .729 , which is financed by the Netherlands Organisation for Scientific Research (NWO). HA acknowledges the support of NWO via a Veni grant. FdG is supported by the VENI research program with project number 639.041.542, which is financed by the Netherlands Organisation for Scientific Research (NWO).

\section{References}

Bernardi, G., Venturi, T., Cassano, R., et al. 2016, MNRAS, 456, 1259 Böhringer, H., Pratt, G. W., Arnaud, M., et al. 2010, A\&A, 514, A32 Bonafede, A., Intema, H. T., Brüggen, M., et al. 2014, MNRAS, 444, L44 Bonafede, A., Cassano, R., Brüggen, M., et al. 2017, MNRAS, 470, 3465 Botteon, A., Shimwell, T. W., Bonafede, A., et al. 2018, MNRAS, 478, 885 Brienza, M., Godfrey, L., Morganti, R., et al. 2017, A\&A, 606, A98 Brüggen, M., Rafferty, D., Bonafede, A., et al. 2018, MNRAS, 477, 3461 Brunetti, G., \& Jones, T. W. 2014, Int. J. Mod. Phys. D, 23, 1430007 Brunetti, G., Setti, G., Feretti, L., \& Giovannini, G. 2001, MNRAS, 320, 365

Brunetti, G., Venturi, T., Dallacasa, D., et al. 2007, ApJ, 670, L5 Brunetti, G., Giacintucci, S., Cassano, R., et al. 2008, Nature, 455, 944

Carilli, C. L., \& Taylor, G. B. 2002, ARA\&A, 40, 319

Cassano, R. 2010, A\&A, 517, A10

Cassano, R., \& Brunetti, G. 2005, MNRAS, 357, 1313

Cassano, R., Brunetti, G., \& Setti, G. 2006, MNRAS, 369, 1577

Cassano, R., Brunetti, G., Setti, G., Govoni, F., \& Dolag, K. 2007, MNRAS, 378, 1565

Cassano, R., Ettori, S., Giacintucci, S., et al. 2010, ApJ, 721, L82

Cassano, R., Brunetti, G., Norris, R. P., et al. 2012, A\&A, 548, A100

Cassano, R., Ettori, S., Brunetti, G., et al. 2013, ApJ, 777, 141

Cassano, R., Brunetti, G., Giocoli, C., \& Ettori, S. 2016, A\&A, 593, A81

Cornwell, T. J., Golap, K., \& Bhatnagar, S. 2005, in Astronomical Data Analysis Software and Systems XIV, eds. P. Shopbell, M. Britton, \& R. Ebert, ASP Conf. Ser., 347, 86

Cuciti, V., Cassano, R., Brunetti, G., et al. 2015, A\&A, 580, A97

Cuciti, V., Brunetti, G., van Weeren, R., et al. 2018, A\&A, 609, A61

Dallacasa, D., Brunetti, G., Giacintucci, S., et al. 2009, ApJ, 699, 1288

de Gasperin, F., Intema, H. T., Shimwell, T. W., et al. 2017, Sci. Adv., 3, e1701634

de Gasperin, F., Dijkema, T. J., Drabent, A., et al. 2019, A\&A, 622, A5 (LOFAR SI)

Donnert, J., Dolag, K., Brunetti, G., \& Cassano, R. 2013, MNRAS, 429, 3564

Eckert, D., Molendi, S., \& Paltani, S. 2011, A\&A, 526, A79

Feretti, L., Giovannini, G., Govoni, F., \& Murgia, M. 2012, A\&ARv, 20, 54

Giacintucci, S., Venturi, T., Cassano, R., Dallacasa, D., \& Brunetti, G. 2009, ApJ, 704, L54

Giovannini, G., Feretti, L., Girardi, M., et al. 2011, A\&A, 530, L5

Golovich, N., Dawson, W. A., Wittman, D. M., et al. 2018, ApJ, submitted [arXiv:1806. 10619]

Hales, S. E. G., Riley, J. M., Waldram, E. M., Warner, P. J., \& Baldwin, J. E. 2007, MNRAS, 382, 1639

Intema, H. T., Jagannathan, P., Mooley, K. P., \& Frail, D. A. 2017, A\&A, 598, A78

Kale, R., Venturi, T., Giacintucci, S., et al. 2013, A\&A, 557, A99

Kale, R., Venturi, T., Giacintucci, S., et al. 2015, A\&A, 579, A92

Kravtsov, A. V., \& Borgani, S. 2012, ARA\&A, 50, 353

Martinez Aviles, G., Ferrari, C., Johnston-Hollitt, M., et al. 2016, A\&A, 595, A116

McMullin, J. P., Waters, B., Schiebel, D., Young, W., \& Golap, K. 2007, in Astronomical Data Analysis Software and Systems XVI, eds. R. A. Shaw, F. Hill, \& D. J. Bell, ASP Conf. Ser., 376, 127

Miley, G. 1980, ARA\&A, 18, 165

Mohr, J. J., Mathiesen, B., \& Evrard, A. E. 1999, ApJ, 517, 627

Molendi, S., \& Pizzolato, F. 2001, ApJ, 560, 194

Murgia, M., Govoni, F., Markevitch, M., et al. 2009, A\&A, 499, 679

Murgia, M., Govoni, F., Feretti, L., \& Giovannini, G. 2010, A\&A, 509, A86

Offringa, A. R., McKinley, B., Hurley-Walker, N., et al. 2014, MNRAS, 444, 606 
Petrosian, V. 2001, ApJ, 557, 560

Piffaretti, R., Arnaud, M., Pratt, G. W., Pointecouteau, E., \& Melin, J.-B. 2011, A\&A, 534, A109

Planck Collaboration XXIX. 2014, A\&A, 571, A29

Reichert, A., Böhringer, H., Fassbender, R., \& Mühlegger, M. 2011, A\&A, 535, A4

Santos, J. S., Rosati, P., Tozzi, P., et al. 2008, A\&A, 483, 35

Savini, F., Bonafede, A., Brüggen, M., et al. 2018, MNRAS, 474, 5023

Savini, F., Bonafede, A., Brüggen, M., et al. 2019, A\&A, 622, A24 (LOFAR SI)

Scaife, A. M. M., \& Heald, G. H. 2012, MNRAS, 423, L30

Sebastian, B., Lal, D. V., \& Pramesh Rao, A. 2017, AJ, 154, 169

Shimwell, T. W., Luckin, J., Brüggen, M., et al. 2016, MNRAS, 459, 277

Shimwell, T. W., Röttgering, H. J. A., Best, P. N., et al. 2017, A\&A, 598, A104

Shimwell, T. W., Tasse, C., Hardcastle, M. J., et al. 2019, A\&A, 622, A1 (LOFAR SI)
Srivastava, S., \& Singal, A. K. 2016, ArXiv e-prints [arXiv:1610.07783] Vallee, J. P., \& Wilson, A. S. 1976, Nature, 259, 451

van Haarlem, M. P., Wise, M. W., Gunst, A. W., et al. 2013, A\&A, 556, A2 van Weeren, R. J., Williams, W. L., Hardcastle, M. J., et al. 2016, ApJS, 223, 2

van Weeren, R. J., Andrade-Santos, F., Dawson, W. A., et al. 2017, Nat. Astron., 1,0005

Venturi, T., Giacintucci, S., Brunetti, G., et al. 2007, A\&A, 463, 937

Venturi, T., Giacintucci, S., Dallacasa, D., et al. 2008, A\&A, 484, 327

Vikhlinin, A., Markevitch, M., Murray, S. S., et al. 2005, ApJ, 628, 655

Webber, J. C. 1974, PASP, 86, 223

Wilber, A., Brüggen, M., Bonafede, A., et al. 2018a, MNRAS, 476, 3415

Wilber, A., Brüggen, M., Bonafede, A., et al. 2018b, MNRAS, 473, 3536

Williams, W. L., van Weeren, R. J., Röttgering, H. J. A., et al. 2016, MNRAS, 460,2385

Xue, Y.-J., \& Wu, X.-P. 2000, ApJ, 538, 65 